\title{
Characteristics of medication overdose presentations to the ED: how do they differ from illicit drug overdose and self-harm cases?
}

\author{
Penny Buykx, ${ }^{1}$ Paul Dietze, ${ }^{2}$ Alison Ritter, ${ }^{3}$ Wendy Loxley ${ }^{4}$
}

${ }^{1}$ Monash University School of Rural Health, Bendigo, Australia ${ }^{2}$ Burnet Institute, Melbourne, Australia

${ }^{3}$ National Drug and Alcohol Research Centre, University of NSW, Sydney, Australia ${ }^{4}$ National Drug Research Institute, Curtin University, Perth, Australia

\section{Correspondence to} Dr Penny Buykx, Monash University School of Rural Health, PO Box 666, Bendigo Central Post Office, VIC 3552 . Australia;

penny.buykx@monash.edu

Accepted 28 May 2009

\section{ABSTRACT \\ Background Medication overdose accounts for $>80 \%$ of hospital presentations for self-harm. Previous research has identified typical characteristics of medication overdose cases; however, these cases have not been well differentiated from other similar presentations, namely (1) illicit drug overdose and (2) self-harm by means other than overdose.}

Method A 12-month audit of medication overdose cases (both intentional and unintentional) attending the emergency department (ED) of a major metropolitan public hospital in Melbourne, Australia was conducted. Comparison was made with patients attending for illicit drug overdose or for self-harm by means other than overdose.

Results Medication overdose cases $(n=453)$ showed a broadly comparable profile with those found in earlier studies (predominantly female gender, aged in their 30 s and referred for psychosocial assessment). A similar though not identical profile was noted for self-harm cases $(n=545)$. In contrast, patients attending for illicit drug overdose $(n=409)$ could be characterised as male, in their 20s and not referred for psychosocial assessment. Illicit drug overdose cases were more likely than either the medication overdose or self-harm cases to be triaged in the most urgent category $(19.3,3.8$ and $3.9 \%$ respectively), suggesting a high level of acuity in this group. However, the illicit drug overdose group on average spent less time in the ED than medication overdose patients, and were less likely to require hospital admission. Conclusion On both demographic and treatment variables, patients attending the ED following a medication overdose more closely resemble those attending for self-harm by means other than overdose than those attending for illicit drug overdose.

\section{BACKGROUND}

Medication overdose or self-poisoning accounts for most emergency department (ED) admissions $(82-95 \%)$ for deliberate self-harm, ${ }^{1-6}$ and $0.3-5.0 \%$ of all ED admissions. ${ }^{178}$ The majority of people presenting to hospital for deliberate self-poisoning are female and aged in their 20 s or 30s. ${ }^{\text {5-7 9-13 }}$ At least $90 \%$ have a diagnosable psychiatric condition, ${ }^{3}{ }^{8}$ most frequently depression, ${ }^{1}{ }^{3} 1014-17$ although substance use disorders, personality disorders, anxiety-related disorders, eating disorders and schizophrenia are also common. ${ }^{3} 91819$ Alcohol has been estimated to be involved in $28-40 \%$ of cases. ${ }^{10} 1320$ Despite being highly recommended, psychosocial assessment of self-poisoning patients occurs in only around half of cases. $^{9}{ }^{21}$
Medication overdose cases generally present from late afternoon to late evening, ${ }^{72} 23$ and are treated more urgently and have longer stays than cases involving other forms of self-injury. ${ }^{57}$ Conservative estimates of repeat presentation to hospital suggest rates of between $12 \%$ and $19 \%$ within 12 weeks to 2 years. ${ }^{711} 13202425$ Individuals with a history of self-poisoning have a greater probability of eventual death by suicide than the general population. ${ }^{26} 27$ In spite of this, those who attend repeatedly for medication overdose are treated less urgently and have shorter stays. ${ }^{7}$

Two Australian health services, the Hunter Area Health Service and the Austin \& Repatriation Medical Centre, have each developed a multidisciplinary response to self-poisoning presentations, including $24 \mathrm{~h}$ toxicological and psychiatric expertise. $^{8}{ }^{28}$ Such models have been argued to provide optimal patient care, and both services have been shown to be associated with a reduced length of hospital stay.

Very few previous studies of medication overdose have compared patients presenting for medication overdose with other relevant patient groups. One study contrasted self-poisoning with other forms of self-inflicted injury ${ }^{5}$ while another compared two forms of self-inflicted harm-trauma and overdose-but did not distinguish between overdoses involving pharmaceutical medications and those involving illicit drugs. ${ }^{29}$ No study has simultaneously compared medication overdose, illicit drug overdose and other forms of self-harm. Such comparison is required because systematic differences between case types need to be taken into account by ED services both when treating individual cases and in the allocation of resources.

In this study we describe the characteristics of medication overdose presentations made to a major inner city public hospital in Melbourne relative to three other patient groups (ie, illicit drug overdose, self-harm by means other than overdose, and the total patient population). We examine the characteristics of cases in the context of management variables and referral patterns with a view to identifying areas in which the ED response could be improved.

\section{METHOD}

We audited all cases attending the St Vincent's Hospital Melbourne ED from 1 November 2003 to 31 October 2004 using the Patient Administration System (PAS)-computerised clinical records completed by ED staff. St Vincent's is an $800+$ bed adult teaching hospital located on the fringe of 
Melbourne's Central Business District (CBD), providing medical, surgical, mental health and other services. The ED is always open. The study was approved by the St Vincent's Human Research Ethics Committee. Three independent groups were identified:

- Medication overdose (MOD)-accidental or deliberate overdoses on medication(s). 'Medication' included any pharmaceutical preparation available on prescription or over-the-counter (OTC), but excluded naturopathic or herbal medications. All cases where the inappropriate (other than prescribed or recommended) consumption of prescription or OTC medications directly contributed to the presentation were audited as 'overdoses'.

- Drug overdose (DOD) - accidental or deliberate intoxication or overdose on illicit $\operatorname{drug}(\mathrm{s})$, that included heroin, amphetamines, ecstasy, GHB ( $\gamma$-hydroxybutyrate), ketamine, cannabis, hallucinogens, cocaine and inhalants. All cases involving recent illicit drug use where the person was substance affected were audited.

- Self-harm (SH) - cases involving thoughts, threats or actions of deliberate self-harm. Suicidal intent was not required for inclusion. Cases where medications or illicit substances were used as the means of self-harm were excluded as they were classified as either MOD or DOD. Types of self-harming behaviour noted or threatened were poisoning by other substances, self-inflicted scratches/cuts/lacerations/stabbing, ingestion of foreign objects, jumping from a height, hanging/ strangulation, deliberate involvement in a car/train/tram accident, exhaust/gassing and burning/electrocution.

A small number of cases fell into both the MOD and DOD categories and were excluded from statistical analysis (see the Results section).

Variables extracted from the PAS included age, sex, triage level assigned to the patient, ${ }^{30}$ day of the week of presentation, time of presentation, time of discharge, discharge status and a unique patient identifier.

Cases were identified by reviewing both the presenting complaint and free text fields on the PAS. The free text field included additional comments by triage and treating staff regarding the presentation. Review of this field increased the likelihood that all relevant cases were included, not just those identified by the presenting complaint. Other information recorded from this field included: alcohol involvement, psychosocial assessment (ie, patient referral to the ED-based mental health and/or social welfare service teams) and whether a security code was called or warned during the presentation (eg, when physical restraint of the patient was required). This information is non-mandatory, meaning that the data are only a minimum estimate of each of these factors. The audit did not involve review of hard copy patient medical records.

\section{Statistical power and analysis}

We estimated that MOD, SH and DOD would, together, comprise $\sim 5 \%$ of the total number of presentations, and hence that 1 year's data would yield $\sim 1500$ events for analysis, comprising approximately equal numbers of the three event types. With 500 events in each group, an RR of 2.0 can be estimated with precision of $61 \%$ relative error, from a prevalence base of $\geq 5 \%$. ${ }^{31}$ We judged that this was sufficient accuracy for our needs, and we therefore included 1 year of data in our sample.

Frequencies of medication misuse or overdose, illicit drug intoxication or overdose, and self-harm presentations were calculated and expressed as a proportion of the ED total.
Multinomial logistic regression was used to examine associations between explanatory variables (age, sex, day, and time of attendance) and patient group. Logistic regression was used to explore the relationship between patient group membership and two binary treatment outcome variables (triage level and discharge destination), using the illicit drug overdose group as the reference group. The ED total was not included in the regression analyses.

\section{RESULTS}

There were 32139 presentations to the ED during the study; 521 cases involving medication misuse or overdose $(1.6 \%$ of all presentations), 477 involving illicit drug intoxication or overdose $(1.5 \%)$ and 732 involving self-harm (2.3\%). Overlap between groups required that independent groups be established for statistical analyses. These were: MOD $(n=453), S H(n=545)$ and DOD $(n=409)$. The 1407 presentations resulted from 1158 individuals $(\mathrm{MOD}=361, \mathrm{SH}=430$ and $\mathrm{DOD}=367)$. Re-presentation rates within the 12 months were: $11.4 \%$ for $\mathrm{MOD}$ (mean $=1.3$, range $1-16) ; 16.3 \%$ for $\mathrm{SH}$ (mean=1.3, range $1-10)$; and $7.4 \%$ for DOD (mean=1.1, range $1-8$ ). These were lower than the $21.4 \%$ re-presentation rate seen for the ED total (mean $=1.4$, range $1-69$ ).

\section{Demographic and temporal characteristics}

While most MOD presentations were female, most $\mathrm{SH}$ and DOD cases were male (table 1). After adjusting for the remaining variables in table 1, females were 3.8 (95\% CI 2.8 to 5.0) times as likely to be in the MOD group and 1.7 (95\% CI 1.3 to 2.2) times as likely to be in the $\mathrm{SH}$ group than the DOD group.

The average age for MOD, $\mathrm{SH}$ and DOD presentations was lower than the ED total (table 1). Overall, DOD cases were younger than the other two groups. When compared with $\mathrm{DOD}$, both $\mathrm{MOD}$ and $\mathrm{SH}$ presentations were less likely to be aged $<20$ (OR 10.0, 95\% CI 5.9 to 16.7 ; and OR 10.2, 95\% CI 5.9 to 16.7 , respectively), less likely to be aged 20-29 (OR 5.9, 95\% CI 4.0 to 9.1 ; and OR 4.9,95\% CI 3.3 to 7.1 , respectively) and less likely to be aged 30-39 (OR 3.2, 95\% CI 2.0 to 5.0; and OR $3.0,95 \%$ CI 2.0 to 4.7 , respectively), after adjusting for the remaining variables in table 1 .

Table 1 Demographic and temporal characteristics of MOD, SH, DOD and total ED presentations

\begin{tabular}{lllll}
\hline & Group & & & \\
\cline { 2 - 5 } Characteristic & $\begin{array}{l}\text { MOD } \\
(\mathbf{n}=\mathbf{4 5 3})\end{array}$ & $\begin{array}{l}\text { SH } \\
(\mathbf{n}=\mathbf{5 4 5 )}\end{array}$ & $\begin{array}{l}\mathbf{D O D} \\
(\mathbf{n}=\mathbf{4 0 9})\end{array}$ & $\begin{array}{l}\text { ED total } \\
(\mathbf{n}=\mathbf{3 2 1 3 9})\end{array}$ \\
\hline Female \% & $63.6^{*}$ & 44.0 & 31.8 & $44.1 \dagger$ \\
Age, mean (SD) & $36.2(13.9)$ & $34.8(12.3)$ & $27.2(8.4)$ & $47.8(21.3) \neq$
\end{tabular}

Age group \%

$\begin{array}{lrrrr}<20 & 6.6 & 6.1 & 17.8 & 4.1 \\ 20-29 & 30.7 & 34.7 & 49.1 & 22.4 \\ 30-39 & 27.2 & 26.4 & 23.5 & 17.2 \\ 40+ & 35.5 & 32.8 & 9.5 & 56.3 \\ \text { Weekday attendance \% } & 68.0 & 71.7 & 59.7 & 70.7 \\ \begin{array}{l}\text { Time of attendance \% } \\ \text { 00:00-05:59 }\end{array} & 19.0 & 16.5 & 26.4 & 13.5 \\ \text { 06:00-11:59 } & 11.5 & 13.9 & 16.9 & 23.5 \\ \text { 12:00-17:59 } & 30.5 & 34.7 & 25.9 & 35.3 \\ \text { 18:00-23:59 } & 39.1 & 34.9 & 30.8 & 27.5\end{array}$

${ }^{*} \mathrm{n}=452$.

$\dagger \mathrm{n}=32083$

$\neq \mathrm{n}=32081$.

DOD, drug overdose; ED, emergency department; MOD, medication overdose; SH, selfharm. 
Initial inspection showed that while $\mathrm{MOD}$ and $\mathrm{SH}$ and total ED presentations were evenly distributed across the week, DOD presentations occurred more frequently on the weekend. After recoding into either 'weekday' (Monday to Friday) or 'weekend' (Saturday and Sunday) presentation (table 1) and adjusting for the remaining variables in table 1 , weekday attendances were 1.4 (95\% CI 1.1 to 1.9$)$ times as likely to be for MOD and $1.7(95 \%$ CI 1.3 to 2.3) times as likely to be for SH than to be for DOD.

The time at which cases presented to the ED was recoded into four 6-hourly blocks. The number of MOD presentations increased throughout the day from $6: 00 \mathrm{~h}$, with the peak occurring between 18:00 and 24:00 h (table 1). A similar pattern was evident for SH and DOD cases, although the peak appeared to be earlier for $\mathrm{SH}$ cases and somewhat later for DOD cases. The MOD group were approximately half as likely to attend as DOD between $24: 00$ and $6: 00 \mathrm{~h}$ (OR $0.6,95 \% \mathrm{CI} 0.4$ to 0.8 ) and between $6: 00$ and $12: 00 \mathrm{~h}$ (OR $0.5,95 \% \mathrm{CI} 0.4$ to 0.8 ). The $\mathrm{SH}$ group were also about half as likely as DOD to attend between 24 and $6: 00$ h (OR $0.6,95 \%$ CI 0.4 to 0.8 ).

\section{Treatment characteristics}

Presentations for the MOD SH, and DOD groups were most commonly given a triage level of ' 3 ' (table 2). Triage level was collapsed into two groups; triage 1 (to be seen immediately) and triage 2-5 (not to be seen immediately). Both the MOD group and $\mathrm{SH}$ group were approximately six times as likely to be categorised as triage 2-5 than DOD (OR 6.1, 95\% CI 3.6 to 10.6 ; and OR 6.0, 95\% CI 3.6 to 9.9, respectively). It therefore seems that more DOD cases were judged to be more urgent than the comparison groups.

The most common discharge destination for all three groups was home (table 2). To investigate whether group membership was predictive of discharge outcomes, those cases who were admitted were compared with those discharged home (and all other cases excluded from the analyses). Compared with DOD cases, MOD and $\mathrm{SH}$ cases were more than three times as likely to be admitted (OR 3.8, 95\% CI 2.5 to 6.3; and OR 3.3, 95\% CI 2.1 to 5.3 , respectively).

For each group a small proportion of people left the department prior to the commencement of treatment $(\mathrm{MOD}=3.8 \%$, $\mathrm{SH}=3.7 \%$ and $\mathrm{DOD}=6.4 \%$ ) and it was not possible to estimate how long such people waited before leaving. For the rest of the

Table 2 Treatment characteristics of MOD, SH, DOD and total ED presentations

\begin{tabular}{|c|c|c|c|c|}
\hline & \multicolumn{4}{|l|}{ Group (\%) } \\
\hline & $\begin{array}{l}\text { MOD } \\
(n=453)\end{array}$ & $\begin{array}{l}\text { SH } \\
(n=545)\end{array}$ & $\begin{array}{l}\text { DOD } \\
(n=409)\end{array}$ & $\begin{array}{l}\text { ED total } \\
(n=32139)\end{array}$ \\
\hline \multicolumn{5}{|c|}{ Triage level (ie, case to be seen...) } \\
\hline 1 (Immediately) & 3.8 & 3.9 & 19.3 & 1.5 \\
\hline $2(\leq 10 \mathrm{~min})$ & 20.1 & 9.4 & 15.6 & 9.3 \\
\hline $3(\leq 30 \mathrm{~min})$ & 63.8 & 44.0 & 39.4 & 40.1 \\
\hline $4(\leq 60 \mathrm{~min})$ & 11.7 & 39.8 & 23.0 & 41.6 \\
\hline $5(\leq 120 \mathrm{~min})$ & 0.7 & 2.9 & 2.7 & 7.5 \\
\hline \multicolumn{5}{|c|}{ Discharge destination } \\
\hline Home & 66.7 & 69.4 & 82.4 & 65.7 \\
\hline Admitted & 21.6 & 19.1 & 6.8 & 24.5 \\
\hline Own risk & 7.5 & 7.2 & 9.1 & 6.0 \\
\hline Another hospital & 2.4 & 2.9 & 1.0 & 2.6 \\
\hline Other* & 1.8 & 1.4 & 1.7 & 1.2 \\
\hline
\end{tabular}

${ }^{*} \mathrm{n}={ }^{\prime}$ Other' discharge destinations included; return to ward, mental health, left after advice, residential care, correctional facility and died in the ED.

$\mathrm{DOD}$, drug overdose; ED, emergency department; MOD, medication overdose; SH, self-harm. cases a calculation was made of the median length of time between being triaged and treatment commencement and between treatment commencement and discharge (table 3).

Analysis of the open text field suggests that more MOD cases involved alcohol than SH or DOD cases (table 4). Patients in the $\mathrm{SH}$ group received the greatest proportion of mental health assessments, while very few DOD cases were referred for such assessment. As recording of these data on the PAS was not mandatory, no statistical comparisons between groups were undertaken.

\section{DISCUSSION}

The contribution made by $\mathrm{MOD}$ cases to the overall $\mathrm{ED}$ patient load $(1.6 \%)$ fell within the range of earlier studies. ${ }^{7} \mathrm{~A}$ similar proportion of cases was accounted for by DOD, although this may have been influenced by the location of the hospital in a busy inner urban area. MOD and DOD presentations, along with those for $\mathrm{SH}$, accounted for almost 1 in every 20 cases attending the hospital, or an average of $4-5$ individuals per day.

In agreement with previous research, ${ }^{72}$ we noted recurrent medication overdose, with $11.4 \%$ of $\mathrm{MOD}$ cases re-presenting for the same reason during the year. However, while DOD recurrence was less frequent, recurrence was more common among the $\mathrm{SH}$ cases and the ED total, meaning that re-presentation is certainly not a unique feature of MOD presentations. Nevertheless, it should be noted that our research cannot exclude the possibility of re-presentation at alternative EDs, re-presentations occurring outside the 12 month time frame and/or overdoses for which help was not sought.

The sex distribution of cases in this study was consistent with previous research. ${ }^{6}{ }^{13}$ However, it should be remembered that all self-harm cases involving medications were assigned to the $\mathrm{MOD}$ group, thereby possibly altering the gender ratio of the $\mathrm{SH}$ group. Further, it should be noted that there were still in excess of 160 men who presented to the ED for MOD in this study.

Consistent with previous research, MOD cases most frequently presented to the $\mathrm{ED}$ in the evenings between 18:00 and 24:00 h, and relatively evenly across different days of the week. $^{72}$ These data are important in terms of resource allocation, with the temporal pattern for all three groups differing from the ED total to at least some extent. As overdose and selfharm cases are likely to require specialist mental health assessment, these findings suggest that ED rosters should ensure the necessary expertise is available overnight and early in the morning. Similarly, toxicological expertise may be in greatest demand at night.

Variation in triage level and the average amount of time spent in the ED may be explained by the differing treatment needs of patients attending for MOD, DOD and SH. MOD cases were triaged more urgently than cases attending the $\mathrm{ED}$ as a whole and spent longer in the department than other patients. Assessment and treatment of these cases may involve several steps (eg, blood tests, administration of antidote), followed by a period of monitoring and supportive care while the person metabolises the medication and, finally, a mental health

Table 3 Median waiting time for MOD, DOD and SH

\begin{tabular}{llll}
\hline & \multicolumn{3}{l}{ Median waiting time h:min } \\
\cline { 2 - 4 } Time point & $\begin{array}{l}\text { MOD } \\
(\mathbf{n = 4 3 6 )}\end{array}$ & $\begin{array}{l}\mathbf{S H} \\
(\mathbf{n}=\mathbf{5 2 5})\end{array}$ & $\begin{array}{l}\text { D0D } \\
(\mathbf{n}=\mathbf{3 8 3})\end{array}$ \\
\hline Triage to treatment commencement & $00: 17$ & $00: 24$ & $00: 09$ \\
Treatment commencement to discharge & $04: 47$ & $01: 56$ & $02: 44$ \\
\hline
\end{tabular}

DOD, drug overdose; MOD, medication overdose; SH, self-harm. 
Table 4 Other information derived from the PAS for MOD, SH and DOD

\begin{tabular}{llll}
\hline & \multicolumn{4}{l}{$\%$ (minimum estimate) } \\
\cline { 2 - 4 } Other information & $\begin{array}{l}\text { MOD } \\
(\mathbf{n = 4 5 3 )}\end{array}$ & $\begin{array}{l}\text { SH } \\
(\mathbf{n = 5 4 5 )}\end{array}$ & $\begin{array}{l}\text { D0D } \\
(\mathbf{n = 4 0 9})\end{array}$ \\
\hline Alcohol related & 24.3 & 17.8 & 17.6 \\
Mental health assessment & 58.9 & 77.2 & 10.0 \\
Social work assessment & 4.4 & 4.0 & 4.2 \\
Security code & 7.9 & 12.3 & 6.1 \\
$\quad$ Actually called & 4.4 & 9.7 & 5.4 \\
Warning & 3.5 & 2.6 & 0.7 \\
\hline
\end{tabular}

DOD, drug overdose; MOD, medication overdose; PAS, Patient Administration System; SH, self-harm.

assessment. The majority of $\mathrm{SH}$ cases in this study did not require medical treatment for an injury, although most did receive a mental health assessment. DOD cases required more immediate attention than the other groups, probably because of the risk of mortality. Nevertheless, DOD cases were infrequently referred for mental health assessment, less likely to be admitted to hospital and left the ED more quickly than the MOD cases.

The rate of assessment found in this study falls short of the universal psychosocial assessment that is recommended following self-harming behaviour, ${ }^{8} 3233$ although the current data may under-report the actual rate of assessments that took place. Some form of mental health or social work assessment was reported to have occurred in $>80 \%$ of the $\mathrm{SH}$ and $>60 \%$ of the MOD cases included in this study. However, it is the low rate of psychosocial assessment of DOD patients that is of greatest concern. The opportunity to intervene at a point of crisis with illicit drug users (ie, when attending the ED following an overdose) was frequently missed, although this group is known to have multiple and complex needs such as high co-morbidity.

Addressing the low rate of psychosocial assessment of overdose cases identified by this study may have significant implications for ED resources. However, the experience of previous health services ${ }^{8} 28$ suggests that a comprehensive response to overdose involving toxicological and psychiatric expertise can result in a net reduction in the length of stay for admitted patients. A worthwhile extension to this study would be to determine how many of those MOD, SH or DOD patients who receive a psychosocial assessment are offered further treatment or referral to other services, the nature of such treatment or referral, and whether or not this offer is taken up by patients. It would also be of interest to explore how patients who attend the ED repeatedly for these conditions are managed, with a view to identifying best practice for reducing the frequency and acuity of presentations.

This study is limited by the exclusion of a small number of cases that could not readily be assigned to one of the three independent groups and the possibility that some eligible cases were missed during the audit of PAS (although every effort was made to be thorough in this regard). The data items identified from the open text field (eg, psychosocial assessment, alcohol use) probably under-represent the true figures as these items are non-mandatory. However, there is no reason to suspect any under-reporting differed systematically between the groups. A full review of medical records may have yielded more complete data, but was beyond the scope and resources of the project. The data are for 12 months only. Ongoing monitoring would allow the stability or otherwise of the findings over time to be examined. Finally, the ED in which the data were collected may not be representative of other hospitals.

\section{CONCLUSION}

The demographic and treatment characteristics of patients attending the ED following a medication overdose more closely resemble patients attending for self-harm (ie, thoughts, threats or actions of self-harm by means other than overdose) than patients attending for illicit drug overdose.

Acknowledgements The late Dr Andrew Dent, former Director of the ED, provided exceptional support for the conduct of the project. Grateful acknowledgement is also made to the ED staff. This work was conducted while PB, PD and AR were all employed at Turning Point Alcohol and Drug Centre, Melbourne, Australia.

Funding The study was partly funded via a grant from beyondblue: the National Depression Initiative. PB was supported by a National Drug Research Institute, Curtin University scholarship. PD and AR are both currently in receipt of a Career Development Award from the NHMRC.

Competing interests None.

Ethics approval This study was conducted with the approval of the St Vincent's Human Research Ethics Committee.

Provenance and peer review Not commissioned; externally peer reviewed.

\section{REFERENCES}

1. Aghanwa H. Attempted suicide by drug overdose and by poison-ingestion methods seen at the main general hospital in the Fiji islands: a comparative study. Gen Hosp Psychiatry 2001;23:266-71.

2. Colman I, Dryden D, Thompson A, et al. Utilization of the emergency department after self-inflicted injury. Acad Emerg Med 2004:11:136-42.

3. Haw C, Hawton K, Houston K, et al. Psychiatric and personality disorders in deliberate self-harm patients. Br J Psychiatry 2001;178:48-54.

4. Hawton K, Fagg J, Simkin S, et al. Trends in deliberate self-harm in Oxford, 1985-1995. Implications for clinical services and the prevention of suicide. Br J Psychiatry 1997;171:556-60.

5. Horrocks J, Price $S$, House A, et al. Self-injury attendances in the accident and emergency department: clinical database study. Br J Psychiatry 2003;183:34-9.

6. Townsend $\mathbf{E}$, Hawton K, Harriss L, et al. Substances used in deliberate self-poisoning 1985-1997: trends and associations with age, gender, repetition and suicide intent. Soc Psychiatry Psychiatr Epidemiol 2001;36:228-34.

7. Taylor D, Cameron P, Eddey D. Recurrent overdose: patient characteristics, habits, and outcomes. J Accid Emerg Med 1998;15:257-61.

8. Whyte I, Dawson A, Buckley N, et al. A model for the management of self-poisoning. Med J Aust 1997;167:142-6.

9. Kapur N, House A, Creed F, et al. Management of deliberate self poisoning in adults in four teaching hospitals: descriptive study. Br Med J 1998;316:831-2.

10. Lo A, Shalansky S, Leung M, et al. Patient characteristics associated with nonprescription drug use in intentional overdose. Can J Psychiatry 2003;48:232-6.

11. Owens D, Wood C, Greenwood D, et al. Mortality and suicide after non-fatal self-poisoning: 16-year outcome study. Br J Psychiatry 2005;187:470-5.

12. Ticehurst S, Carter G, Clover K, et al. Elderly patients with deliberate self-poisoning treated in an Australian general hospital. Int Psychogeriatr 2002;14:97-105.

13. Vaiva G, Ducrocq F, Meyer P, et al. Effect of telephone contact on further suicide attempts in patients discharged from an emergency department: randomised controlled study. Br Med J 2006;332:1241-5.

14. Hagedorn J, Omar $\mathrm{H}$. Retrospective analysis of youth evaluated for suicide attempt or suicidal ideation in an emergency room setting. Int $J$ Adolesc Med Health 2002;14:55-60.

15. Kingsbury S, Hawton K, Steinhardt K, et al. Do adolescents who take overdoses have specific psychological characteristics? A comparative study with psychiatric and community controls. J Am Acad Child Adolesc Psychiatry 1999;38:1125-31.

16. Lifshitz M, Gavrilov V. Deliberate self-poisoning in adolescents. Isr Med Assoc J 2002;4:252-4.

17. Matthews JD, Fava M. Risk of suicidality in depression with serotonergic antidepressants. Ann Clin Psychiatry 2000;12:43-50.

18. Carter G, Issakidis C, Clover K. Correlates of youth suicide attempters in Australian community and clinical samples. Aust N Z J Psychiatry 2003;37:286-93.

19. Kapur N, Cooper J, Hiroeh U, et al. Emergency department management and outcome for self-poisoning: a cohort study. Gen Hosp Psychiatry 2004;26:36-41

20. Carter G, Clover K, Whyte I, et al. Postcards from the EDge project: randomised controlled trial of an intervention using postcards to reduce repetition of hospital treated deliberate self poisoning. BMJ 2005;331:805.

21. Kapur N, House A, Creed F, et al. General hospital services for deliberate selfpoisoning: an expensive road to nowhere? Postgrad Med J 1999;75:599-602.

22. Buckley N, Whyte I, Dawson A. There are days... and moons ... and public holidays. Self-poisoning is not lunacy. Med J Aust 1993;159:786-9.

23. Heale $\mathbf{P}$, Dietze $\mathrm{P}$, Cvetkovski S. Benzodiazepine related ambulance attendance in Melbourne. VAADA Annual Conference - Everyday Drugs, Everyday Problems. Carlton, Melbourne, 2002

24. Carter G, Whyte I, Ball K, et al. Repetition of deliberate self-poisoning in an Australian hospital-treated population. Med J Aust 1999;170:307-11. 
25. Kapur $\mathbf{N}$, House A, Dodgson K, et al. Effect of general hospital management on repeat episodes of deliberate self-poisoning: cohort study. Br Med J 2002;325:866-7.

26. Carter G, Reith D, Whyte I, et al. Non-suicidal deaths following hospital-treated self-poisoning. Aust N Z J Psychiatry 2005;39:101-7.

27. Reith D, Whyte I, Carter G, et al. Risk factors for suicide and other deaths following hospital treated self-poisoning in Australia. Aust N Z J Psychiatry 2004:38:520-5.

28. Lee V, Kerr JF, Braitberg G, et al. Impact of a toxicology service on a metropolitan teaching hospital. Emerg Med 2001;13:37-42.

29. Taylor D, Cameron P. Deliberate self-inflicted trauma: population demographics, the nature of injury and a comparison with patients who overdose. Aust N Z J Public Health 1998;22:120-5.
30. Australasian College for Emergency Medicine. Policy on the Australasian Triage Scale. 2000. http://www.acem.org.au/media/policies_and_guidelines/

P06 Aust Triage Scale - Nov 2000.pdf (accessed $\overline{1} 4$ July 2004).

31. Clayton $\overline{\mathbf{D}}$, Hills $\overline{\mathrm{M}}$. Statistical models in epidemiology. New York: Oxford University Press, 1993

32. Hawton K. General hospital management of suicide attempters. In: Hawton K, van Heeringen C, eds. The international handbook of suicide and attempted suicide. Chichester, UK: John Wiley \& Sons Ltd, 2000:519-37.

33. Hickey L, Hawton K, Fagg J, et al. Deliberate self-harm patients who leave the accident and emergency department without a psychiatric assessment: a neglected population at risk of suicide. J Psychosom Res 2001;50:87-93. 